\title{
PROKLA-Redaktion
}

\section{Editorial: Peripherer Kapitalismus in Europa}

Mit dem Ende der Sowjetunion und der Auflösung des von ihr beherrschten Staatenblocks schien für die Länder Osteuropas der Weg frei, um aus der realsozialistischen "zweiten" in die kapitalistisch „erste“ Welt zu gelangen und dort von Wohlstand und Demokratic zu profitieren. So wünschte es sich jedenfalls die Mehrheit der Bevölkerung der mittelund osteuropäischen Länder und so verkündeten es auch westliche Politiker und Wirtschaftswissenschaftler: mit der schnellen Einführung marktwirtschaftlicher Strukturen, der Privatisierung der staatlichen Unternehmen und der Einrichtung parlamentarisch-demokratischer Institutionen sollte das nach dem Zusammenbruch des Realsozialismus unausweichliche „Tal der Tränen" bald durchschritten sein. „Blühende Landschaften", die einst ein Kanzler Kohl den Menschen der DDR versprochen hatte, wurden nicht nur dort erwartet.

Zwar wurden in allen ehemals, sozialistischen“ Ländern marktwirtschaftliche Reformen eingeführt, doch haben die neuen osteuropäische Kapitalismen - trotz der historisch einmaligen Radikalität bei der Einführung von Privatisierungs-, Liberalisierungs- und Deregulierungsmaßnahmen - noch immer recht wenig Ähnlichkeiten mit ihren westlichen Vorbildern. Ende der 1990 Jahre reicht die wirtschaftliche Leistungsfähigkeit (gemessen im Bruttoinlandsprodukt pro Kopf) der meisten osteuropäischen EU-Beitrittskandidaten nicht einmal an diejenige der ärmeren EU Länder wie Griechen- land heran - und diese Beitrittskandidaten bilden innerhalb Osteuropas Länder den Wohlstandsgürtel. Entscheidender noch als das absolute Entwicklungsgefälle ist aber dessen Dynamik: Der Abstand zwischen West- und Osteuropa hat sich in den 1990er Jahren vergrößert und nicht verringert; die ungleiche Entwicklung hat sich deutlich verstärkt.

Für diese - bislang jedenfalls - magere Bilanz nach 12 Jahren Übergang zu Demokratie und Marktwirtschaft hat die Transformationsforschung, die zu Anfang der 1990er Jahre propagierte, dass eine schnelle und umfassende Einführung marktwirtschaftlicher Basisinstitutionen fast im Selbstlauf ökonomische Effizienz und Wohlstand generieren würde, wenig überzeugende Erklärungen zu bieten. Es wird immer deutlicher, dass die Kombination aus sozialistischen Hinterlassenschaften und neuen marktwirtschaftlich-demokratischen Institutionen einen Abstieg in die dritte Welt keineswegs ausschließt. Ökonomische Rückständigkeit, schwache Transformationsstaaten und die hohe Bedeutung von Kapitalimporten für die (partielle) Modernisierung lassen zudem Erinnerungen an die osteuropäischen Kapitalismen an der Wende vom 19. zum 20. Jahrhundert aufkommen. War also der Sozialismus nichts weiter als ein „Umweg von der Peripherie in die Peripherie“ (Ivan T. Berend)? Grund genug, nach dem „Zwischenspiel" des Sozialismus, welches die Abhängigkeit Osteuropas von Weltmarktzusammenhängen und von kapitalistisch. 
imperialistischen Mächten für kurze Zeit reduziert hatte, erneut über peripheren Kapitalismus in Europa nachzudenken. Die kritische Transformationsforschung hat diesen Begriff längst zur Analyse der osteuropäischen Entwicklungen und Verwicklungen genutzt. Allerdings geht sie zum Teil recht sorglos mit Begriffen wie Peripherie oder Hinterhof um. Der Verdacht, Osteuropa würde erneut in das Muster einer kolonialen Arbeitsteilung gepresst, bestätigt sich keinesfalls so umstandslos. Zwar hat sich Russland in der Tat in einen typischen "Erdölstaat" (Terry Lynn Karl) verwandelt, und einige zentralasiatische Republiken leben ausschließlich vom "weißen Gold", der Baumwolle. Je „europäischer" die Peripherie, desto komplexer jedoch ihre Form der Einbindung in die internationale Arbeitsteilung: Während z.B. Rumänien hauptsächlich von der Lohnveredelung in der Textil- und Bekleidungsindustrie lebt, hat sich Ungarn in den letzten Jahren zu einem „High-tech“ Exporteur in die EU gemausert. Diese Differenzierung weist darauf hin, dass $\mathrm{Ka}$ tastrophen- und Abhängigkeitsszenarien nicht für jedes osteuropäische Land im gleichen Masse zutreffend sind. Gegen die allzu simple Peripherisierungsthese spricht auch, dass die Transformationsstaaten keineswegs ,unschuldige Opfer" abstrakter Weltmarkttendenzen oder neoimperialistischer Mächte sind: Vielmehr sind es die Transformationseliten und -gesellschaften selber, die auf eine schnelle Rückkehr in den kapitalistischen Weltmarkt und auf die Aufnahme in internationale Organisationen drängen. Eine empirisch und theoretisch fundierte Bestimmung dessen, was Peripherisierung im osteuropäischen Kontext heißt, ist daher zentrales Anliegen dieses Heftes.

Eine genauere Bestimmung der europäischen Peripherie ist allerdings unmöglich, ohne einen Blick auf die Veränderungen im Zentrum zu nehmen. Prozesse der neoliberalen Restrukturierung des globalen Kapitalismus haben nicht nur zu einer Transformation der Nationalstaaten in den "nationalen Wettbewerbsstaat" (Joachim Hirsch) geführt, sondern zugleich, wie neuere neogramscianische und regulationstheoretische Untersuchungen deutlich machen, regionale politische Reorganisationsprozesse initiiert, die in transnationalen Herrschaftsprojekten münden. Die EU ist offensichtlich das am weitesten fortgeschrittene regionale Herrschaftsprojekt, und gleichzeitig von zentraler Bedeutung für Osteuropa. Dorothec Bohle untersucht in ihrem Beitrag die Agenda einer sich herausbildenden transnationalen kapitalistischen Klasse. Sie argumentiert, dass sowohl die Vertiefung der europäischen Integration seit Mitte der 1980er Jahre wie auch die Erweiterung in hohem Maße auf deren Interessen zurückgeht. Während die Vertiefung jedoch in ein prekäres hegemoniales Projekt münden konnte, in dem durch finanzielle Transferleistungen und den Appell an nationale Gefühle auch subalterne Klassen und Positionen eingebunden wurde, beruht die konkrete Form der Einbindung Osteuropas seit Anfang der 1990er Jahre auf dem Export einer sehr viel "marktradikaleren" und weniger konsensualen Variante des Neoliberalismus. Dies erlaubt transnationalen Kapitalgruppen den Zugriff auf die osteuropäischen Standorte, und schützt die EU gleichzeitig vor zu heftigen internen verteilungspolitischen Konflikten.

Dass Peripherisierung keineswegs ein einheitlicher Prozess ist, sondern differenziert betrachtet werden muss, zeigt der Beitrag von Gisela Neunhöffer und Anne Schüttpelz, die zwei unterschiedliche Formen der Peripherisierung unterscheiden: Auf der einen Seite stehen die EU-Beitrittskandidaten, deren Transformationsprozesse in hohem Maße durch die EU-Erweiterungspolitik kontrolliert und gesteuert wird. Die EU-Einbindung schafft verlässliche Bedingungen für das Engagement ausländischen Kapitals und erlaubt damit eine außengesteuerte par- 
tielle Modernisierung. Auf der anderen Seite stehen die Länder, denen diese Perspektive nicht offen steht. Deren Weltmarktintegration erweist sich als offener und fragmentierter. Verwertungsbedingungen für in- und ausländisches Kapital bleiben prekär, die Integration in transnationale Produktionsnetzwerke eher die Ausnahme als die Regel. Es scheint also, dass eine transnational-abhängige Integration in den EU-Raum immer noch eine bessere Form der Dependenz ist als eine direlkte Konfrontation mit dem Welimarkt.

Wie sieht es aber mit den Chancen der osteuropäischen Länder aus, innerhalb der EU einen Aufholprozeß zu initiieren? Dieser Frage geht Otto Holman in einem Vergleich der anstehenden Osterweiterung der EU mit der früheren Süderweiterung nach. Er argumentiert, dass im Gegensatz zu Spanien (und in geringerem Maße zu Portugal und Griechenland), denen in der Tat seit ihrer EUMitgliedschaft die erfolgreiche Integration in das kapitalistische Kernland gelungen ist, Osteuropa absehbar einen peripheren Status beibehalten wird. Sowohl interne Faktoren, insbesondere das FehIen potentiell mächtiger endogener kapitalistischer Akteure, wie externe Faktoren, insbesondere die sich verringernde finanzielle Solidarität der EU mit den Neumitgliedern mindern Osteuropas Chancen auf ein erfolgreiches Aufholen.

Während Holman den EU-Akteuren eine bestimmende Rolle in der Gestaltung der osteuropäischen Entwicklungspfade zuweist, warnen Nicole Lindstrom und Dóra Piroska vor einer zu schnellen Verallgemeinerung dieses Tatbestandes. Am slowenischen Beispiel zeigen sie, dass die osteuropäischen Beitrittskandidaten trotz der Machtfülle der EU und des transnationalen Kapitals durchaus Handlungsspielräume in der Umsetzung der europäischen Vorgaben haben. Slowenien wiedersetzte sich bislang erfolgreich einer Durchdringung (nicht nur) seiner strategischen Sektoren durch ausländisches
Kapital. Die Tatsache, dass Slowenien zugleich zu den erfolgreichsten Transformationsländern gehört, lässt natürlich die Fragen aufkommen, warum nicht mehr Staaten dem "slowenischen Weg" gefolgt sind, ob er tatsächlich einen Weg aus der Peripherie weist, und wie dauerhaft dieser Sonderweg sein kann.

Petra Schaper-Rinkel geht in ihrem Beitrag der Frage, wie die Agenda der EU die konkrete Form der Erweiterung prägt, am Beispiel der europäischen Informationsgesellschaft nach. Sie zeigt, wie die EU mit ihren scheinbar rein technischen Vorgaben für Osteuropa zugleich eine hierarchische Arbeitsteilung vorbereitet, die es den (vor allem westeuropäischen) Unternehmen erlaubt, ihre Produktionsnetzwerke zu reorganisieren und damit ihre weltweite Wettbewerbsfähigkeit zu steigern. Osteuropäische Beitrittskandidaten, oder auch westliche Akteure mit anderen technologiepolitischen Vorstellung haben wenig Chancen, den politischen Gehalt der EU-Vorgaben zu problematisieren.

Die Befunde dieses Hefts deuten insgesamt auf den Nutzen des PeripherieKonzeptes für Osteuropa hin. Für wie gesichert aber kann unser Wissen über den gegenwärtigen Kapitalismus in Osteuropa und über das sozialistische System überhaupt gelten? Béla Greskovits zeigt in seinem Artikel am Beispiel der Suche nach dem "wahren" sozialistischen Bruttosozialprodukt, wie sehr der Druck im Wissenschaftssystem, die Dinge voranzutreiben, das Verfolgen professioneller Ambitionen und die Geschwindigkeit, die durch Beratertätigkeiten diktiert wird, das theoretische und empirische Verständnis des osteuropäischen Sozialismus und Kapitalismus beeinflussen. Obwohl die Größe des "sozialistischen Bruttosozialprodukts" seit Anfang der 1990er Jahre einer ständigen Revision unterworfen wurde, kann man keineswegs von einem Lernprozeß über die wahre Natur des sozialistischen Systems sprechen. Viel- 
mehr führt vorschnelle Theoriebildung auf empirisch ungesicherter Grundlage zu der Entstehung einer neuen Welt der "Fakten", welche die hoffnungslose Rückständigkeit der realsozialistischen Länder als einzig legitime Interpretation erscheinen lassen. Während vor 1989 die Stabilität des Realsozialismus und eine wirtschaftliche Leistungsfähigkeit, die zumindest in die Nähe des Niveaus der westlichen Länder reichte, weitgehend unbestritten waren, wird der (früher unvorstellbare) Zusammenbruch im Nachhinein zum sozusagen naturnotwendigen, selbstverständlichen Ereignis.

Während sich der Heftschwerpunkt mit der gegenwärtigen Herausbildung des Kapitalismus in Osteuropa beschäftigt, untersucht Peter Kriedte in einem Beitrag außerhalb des Schwerpunkts eine Frage der historischen Herausbildung des Kapitalismus: ob und in welcher Hinsicht man von einer dem industriellen Kapitalismus vorgelagerten eigenständi- gen Phase des "Handelskapitalismus“ ausgehen kann. Einige Andeutungen dazu finden sich bereits bei Marx, während etwa Fernand Braudel eine derartige Strukturierung der Geschichte rundweg ablehnt. In dem Beitrag wird versucht, einige Elemente einer Theorie des Handelskapitalismus zu skizzieren. Dabei wird auch deutlich, dass der Übergang vom Handelskapitalismus zum Industriekapitalismus keineswegs einer historischen Notwendigkeit folgt, sondern von einer Reihe historisch kontingenter Faktoren abhängig ist: auch in der Vergangenheit war Geschichte ein offener Prozess.

Der besondere Dank der Redaktion gilt Dorothee Bohle, die nicht nur einen Artikel zu dem Heft beisteuerte, sondern als Gastredakteurin die vorliegende Ausgabe auch konzipiert und mit viel Engagement betreut hat.

\section{PROKLA 130 (März 2003): Gewerkschaften ... zur Sonne, zur Freiheit?}

Angesichts von Globalisienung, Standortkonkurrenz und Massenarbeitslosigkeit sind die traditionellen Formen der gewerkschaftlichen Interessenvertretung immer fragwürdiger geworden. Allerdings konnten bisher weder der Übergang zum Co-Management noch die herkömmlichen Formen linker Betriebspolitik überzeugende Alternativen aufzeigen. Auch die Ergebnisse korporativer Politikformen, etwa im Rahmen des "Bündnisses für Arbeit" haben nicht den von manchen erhofften Durchbruch in Richtung höherer Beschäftigung gebracht. Gleichzeitig leiden Gewerkschaften an Mitgliederschwund und sind insbesondere für jüngere ArbeitnehmerInnen wenig attraktiv. Die Gewerkschaften haben Schwierigkeiten, mit veränderten Lebensverhältnissen und Werthaltungen umzugehen: starre Organisationsstrukturen und mangelnde innerorganisatorische Demokratie sind selbst produzierte Bestandteile der gewerkschaftlichen Krise. Die Reaktionen auf diese Situation fallen in einzelnen Ländern recht unterschiedlich aus, sie reichen von Gewerkschaftsfusionen in Deutschland bis zu gewerkschaftlichen Neugründungen wie SUD in Frankreich oder den Basisaktivitäten der Cobas in Italien. Mit neuen Politikformen bemühen sich Gewerkschaften um die Mobilisierung von KonsumentInnen, um auf diese Weise Unternehmen zu einer Verbesserung der Arbeitsbedingungen zu zwingen. Auch sind schon erste Kooperationen zwischen Gewerkschaften und globalisierungskritischer Bewegung entstanden. Diese Umbrüche und die veränderten gewerkschaftlichen Praxisformen sollen auf nationaler wie internationaler Ebene untersucht werden. 